\title{
Shannon versus Kullback-Leibler Entropies in Nonequilibrium Random Motion
}

\author{
Piotr Garbaczewski* \\ Institute of Physics, University of Zielona Góra, PL 65-516 Zielona Góra, Poland
}

October 16, 2018

\begin{abstract}
We analyze dynamical properties of the Shannon information entropy of a continuous probability distribution, which is driven by a standard diffusion process. This entropy choice is confronted with another option, employing the conditional Kullback-Leibler entropy. Both entropies discriminate among various probability distributions, either statically or in the time domain. An asymptotic approach towards equilibrium is typically monotonic in terms of the Kullback entropy. The Shannon entropy time rate needs not to be positive and is a sensitive indicator of the power transfer processes (removal/supply) due to an active environment. In the case of Smoluchowski diffusions, the Kullback entropy time rate coincides with the Shannon entropy "production" rate.
\end{abstract}

PACS numbers: 02.50.-r, 05.40-a, 89.70.+c

We consider diffusion processes in $R$ with a constant or time-dependent diffusion coefficient $D$, characterized (non-uniquely) by space-time inhomogeneous probability densities $\rho=\rho(x, t)$ and local velocity fields (drifts or currents). We specify the diffusive dynamics to refer to of a mass $m$ particle in the external field of force, here taken to be conservative: $F=F(x)=-\nabla V$. The associated Smoluchowski diffusion process with a forward drift $b(x)=\frac{F}{m \beta}$ is analyzed in terms of the FokkerPlanck equation for the probability density $\rho(x, t),[1,2,3]$ :

$$
\partial_{t} \rho=D \triangle \rho-\nabla(b \cdot \rho)
$$

with the initial data $\rho_{0}(x)=\rho(x, 0)$. We assume a priori the Einstein fluctuation-dissipation relationship $D=\frac{k_{B} T}{m \beta}$, where $\beta$ is interpreted as the friction (damping) parameter, $T$ is the temperature of the bath, $k_{B}$ being the Boltzmann constant .

With a solution $\rho(x, t)$ of the Fokker-Planck equation, we associate the Shannon information entropy of a continuous probability distribution, also named differential entropy, 4, 5] 6]:

$$
\mathcal{S}(t)=-\int \rho(x, t) \ln \rho(x, t) d x
$$

which is typically not a conserved quantity. The rate of change in time of $\mathcal{S}(t)$ readily follows.

${ }^{*}$ Corresponding author: p.garbaczewski@if.uz.zgora.pl 
Anticipating further discussion, let us stress that even in case of plainly irreversible diffusive dynamics, it is by no means obvious that the differential entropy should grow, decay (diminish) or show up a mixed behavior. Normally one tries, in terms of a suitable entropy definition, to justify the popular albeit tacit assumption that "generically" the time rate of "entropy" (whatever it is, be that $\dot{\mathcal{S}}>0)$ is non-negative.

We take for granted the validity of standard assumptions which allow to interchange derivatives with indefinite integrals. Boundary restrictions upon $\rho, v \rho$ and $b \rho$ to vanish at spatial infinities (or at finite spatial volume boundaries) yield the rate equation:

$$
\frac{d \mathcal{S}}{d t}=\int\left[\rho(\nabla \cdot b)+D \cdot \frac{(\nabla \rho)^{2}}{\rho}\right] d x
$$

which in case of $b=0$ refers to the one-dimensional free Brownian motion (Wiener process), when $\dot{\mathcal{S}}>0$. The trivial example is provided by the familiar heat kernel $\rho(x, t)=(4 \pi D t)^{-1 / 2} \exp \left(-x^{2} / 4 D t\right)$ whose differential entropy reads $\mathcal{S}(t)=(1 / 2) \ln (4 \pi e D t)$, implying $\dot{\mathcal{S}}=1 / 2 t>0$.

We can rewrite Eq. (3) in a number of equivalent ways. To this end let us introduce the notion of the current velocity of Brownian particles in external force field (with a short-hand notation $v \doteq$ $v(x, t)): v \doteq b-u=\frac{F}{m \beta}-D \frac{\nabla \rho}{\rho}$ which allows us to transform the Fokker-Planck equation into the continuity equation $\partial_{t} \rho=-\nabla(v \cdot \rho)$ and back. Since $\left\langle u^{2}\right\rangle=-D\langle\nabla \cdot u\rangle$, we have: $D \dot{\mathcal{S}} \doteq$ $D\langle\nabla \cdot b\rangle+\left\langle u^{2}\right\rangle=D\langle\nabla \cdot v\rangle=-\langle v \cdot u\rangle$.

By reasons to become clear in below, we shall pay particular attention to the entropy balance equation in the form:

$$
D \dot{\mathcal{S}}=\left\langle v^{2}\right\rangle-\langle b \cdot v\rangle
$$

where $\langle\cdot\rangle$ denotes the mean value with respect to $\rho$.

This balance equation is extremely persuasive when recast in the thermodynamically motivated form:

$$
\frac{d \mathcal{S}}{d t}=\left(\frac{d \mathcal{S}}{d t}\right)_{i n}-\frac{d \mathcal{Q}}{d t} .
$$

The non-negative term $(\dot{\mathcal{S}})_{\text {in }}=(1 / D)\left\langle v^{2}\right\rangle$ in Eq. (4) may be interpreted as the measure of time rate at which the Shannon information entropy of the process shows a tendency to grow (is generated due to an implicit thermal agitation), at the expense of the environment. This excess "entropy production" by the environment may possibly be counterbalanced by the entropy/heat removal due to dissipation, provided we have $\dot{\mathcal{Q}}>0$. The characteristic "power release" expression:

$$
\frac{d \mathcal{Q}}{d t} \doteq \frac{1}{D} \int \frac{1}{m \beta} F \cdot j d x=\frac{1}{D}\langle b \cdot v\rangle
$$

in the formal thermodynamical lore is expected to refer to the time rate at which the mechanical work per unit of mass is dissipated (returned back to the reservoir) in the form of heat. Notice that $k_{B} T \dot{\mathcal{Q}}=\int F \cdot j d x$, where $b=F /(m \beta), j=v \rho$ and $T$ stands for the temperature of the bath. 
However, in the diffusion context, $\dot{\mathcal{Q}}$ has an unspecified sign. It may be positive, with an interpretation of the power absorption by the environment. $\dot{\mathcal{Q}}$ may take negative values, when the power is drained out from the environment. In below we shall discuss those issues for the exemplary Ornstein-Uhlenbeck process.

Of particular interest is the case of a constant information entropy $\dot{\mathcal{S}}=0$ which amounts to the existence of steady states, $[8$. In the simplest case, when the diffusion current vanishes, we encounter the primitive realization of the state of equilibrium with an invariant density $\rho$. Then, $b=u=D \nabla \ln \rho$ and we readily arrive at the classic equilibrium identity for the Smoluchowski process:

$$
-\left(1 / k_{B} T\right) \nabla V=\nabla \ln \rho
$$

which determines the functional form of the invariant density in case of a given conservative force field, 11, 7]. There is an ample discussion in Ref. [8] of how these properties match with time reversal of the stationary diffusion process and the vanishing of the entropy production rate $(\dot{\mathcal{S}})_{\text {in }}$.

Coming back to the general discussion, let us define the so-called (fictitious) thermodynamic force $F_{t h} \doteq v / D$ associated with the Smoluchowski diffusion and introduce its corresponding timedependent potential function $\Psi(x, t)$ :

$$
k_{B} T F_{t h}=F-k_{B} T \nabla \ln \rho \doteq-\nabla \Psi .
$$

Notice that $v=-(1 / m \beta) \nabla \Psi$. In the absence of external forces (free Brownian motion), we obviously get $F_{t h}=-\nabla \ln \rho=-(1 / D) u$.

The mean value of the potential

$$
\Psi=V+k_{B} T \ln \rho
$$

associates with the diffusion process an obvious analogue of the thermodynamical Helmholtz free energy:

$$
\langle\Psi\rangle=\langle V\rangle-T \mathcal{S}_{G}
$$

where the dimensional (Gibbs-type) version $\mathcal{S}_{G} \doteq k_{B} \mathcal{S}$ of the information entropy has been introduced. The expectation value of the mechanical force potential $\langle V\rangle$ plays here the role of (mean) internal energy.

By assuming that $\rho V v$ vanishes at integration volume boundaries (or infinity), we easily get the time rate of Helmholtz free energy at a constant temperature $T$ :

$$
\frac{d}{d t}\langle\Psi\rangle=-k_{B} T \dot{\mathcal{Q}}-T \dot{\mathcal{S}}_{G}
$$

By employing Eq. (5), we readily arrive at

$$
\frac{d}{d t}\langle\Psi\rangle=-\left(k_{B} T\right)\left(\frac{d \mathcal{S}}{d t}\right)_{i n}=-(m \beta)\left\langle v^{2}\right\rangle
$$


which either identically vanishes (equilibrium) or remains negative.

Thus, Helmholtz free energy either remains constant in time or decreases as a function of time at the rate set by the information entropy "production" $\dot{\mathcal{S}}_{i n}$. One may expect that actualy $\langle\Psi\rangle(t)$ drops down to a finite minimum as $t \rightarrow \infty$.

However, this feature is a little bit deceiving. One should be aware that a finite minimum as well may not exist, which is the case e.g. for the free Brownian motion. Also, non-unique minima need to be excluded as well.

Till now, we have deliberately avoided any use of the relative Kullback-Leibler entropy, [5, 6, 7], which is often invoked for a comparison purpose to tell "how far from each other" two probability densities are. However, a reliability of the Kullback entropy may be questioned: this entropy fails to quantify properly a "comparison" of once given density function to itself, if considered at different instants of its non-stationary time evolution.

To analyze defective features of the Kullback entropy in a fully controllable way, let us consider a one parameter family of Gaussian densities $\rho_{\alpha}=\rho(x-\alpha)$, with the mean $\alpha \in R$ and the standard deviation fixed at $\sigma$. They share the very same value $\mathcal{S}_{\sigma}=\frac{1}{2} \ln \left(2 \pi e \sigma^{2}\right)$ of the Shannon information (differential) entropy, independent of $\alpha$.

If we admit $\sigma$ to be an additional free parameter, a two-parameter family of Gaussian densities $\rho_{\alpha} \rightarrow \rho_{\alpha, \sigma}(x)$ appears. Such densities, corresponding to different values of $\sigma$ and $\sigma^{\prime}$ do admit an "absolute comparison" in terms of the Shannon entropy:

$$
\mathcal{S}_{\sigma^{\prime}}-\mathcal{S}_{\sigma}=\ln \left(\frac{\sigma^{\prime}}{\sigma}\right)
$$

and the outcome is insensitive to translation parameters $\alpha$ and $\alpha^{\prime}$.

By denoting $\sigma \doteq \sigma(t)$ and $\sigma^{\prime} \doteq \sigma\left(t^{\prime}\right)$ we can make any density amenable to the "absolute comparison" formula at different time instants $t^{\prime}>t>0$. For the heat kernel we have $\sigma(t)=\sqrt{2 D t}$ and therefore $\left(\sigma^{\prime} / \sigma\right)=\sqrt{t^{\prime} / t}$.

There are many inequivalent ways to evaluate the "divergence" or "convergence" between probability distributions. The relative (Kullback) entropy is typically used to quantify such divergence relative to the prescribed reference density, [7].

We define the Kullback entropy $\mathcal{K}\left(\theta, \theta^{\prime}\right)$ for a one-parameter family of probability densities $\rho_{\theta}$, so that the "distance" between any two densities in this family can be directly evaluated. Let $\rho_{\theta^{\prime}}$ denote the reference probability density. We have, [5, 6]:

$$
\mathcal{K}\left(\theta, \theta^{\prime}\right) \doteq \mathcal{K}\left(\rho_{\theta} \mid \rho_{\theta^{\prime}}\right)=\int \rho_{\theta}(x) \ln \frac{\rho_{\theta}(x)}{\rho_{\theta^{\prime}}(x)} d x .
$$

which, in view of the concavity of the function $f(w)=-w \ln w$, is positive.

Let us indicate that the negative of $\mathcal{K}, \mathcal{H}_{c} \doteq-\mathcal{K}$, named the conditional entropy, is predominantly used in the literature [6, 9, 7] because of its affinity (regarded as a formal generalization) to the 
differential entropy. Then e.g. one investigates an approach of $-\mathcal{K}$ towards its maximum (usually achieved at the value zero) when a running density is bound to have a unique stationary asymptotic, [9].

If we take $\theta^{\prime} \doteq \theta+\Delta \theta$ with $\Delta \theta \ll 1$, the following approximate formula holds true under a number of standard assumptions:

$$
\mathcal{K}(\theta, \theta+\Delta \theta) \simeq \frac{1}{2} \mathcal{F}_{\theta} \cdot(\Delta \theta)^{2}
$$

where $\mathcal{F}_{\theta}$ denotes so-called Fisher information measure. With this proviso, we can evaluate the Kullback distance within a two-parameter $(\alpha, \sigma)$ family of Gaussian densities, by taking $\theta \rightarrow \alpha$.

Passing to $\alpha^{\prime}=\alpha+\Delta \alpha$ at a fixed value of $\sigma$ we arrive at:

$$
\mathcal{K}(\alpha, \alpha+\Delta \alpha) \simeq \frac{(\Delta \alpha)^{2}}{2 \sigma^{2}} .
$$

For the record, we note that the respective Shannon entropies do coincide: $\mathcal{S}_{\alpha}=\mathcal{S}_{\alpha+\Delta \alpha}$.

Analogously, we can proceed with respect to the label $\sigma$ at $\alpha$ fixed:

$$
\mathcal{K}(\sigma, \sigma+\Delta \sigma) \simeq \frac{(\Delta \sigma)^{2}}{\sigma^{2}}
$$

when, irrespective of $\alpha$ :

$$
\mathcal{S}_{\sigma+\Delta \sigma}-\mathcal{S}_{\sigma} \simeq \frac{\Delta \sigma}{\sigma}
$$

By choosing $\theta \rightarrow \sigma^{2}$ at $\alpha$ fixed, we get (now the variance $\sigma^{2}$ is modified by its increment $\Delta\left(\sigma^{2}\right)$ ):

$$
\mathcal{K}\left(\sigma^{2}, \sigma^{2}+\Delta\left(\sigma^{2}\right)\right) \simeq \frac{\left[\Delta\left(\sigma^{2}\right)\right]^{2}}{4 \sigma^{4}}
$$

while

$$
\mathcal{S}_{\sigma^{2}+\Delta\left(\sigma^{2}\right)}-\mathcal{S}_{\sigma^{2}} \simeq \frac{\Delta\left(\sigma^{2}\right)}{2 \sigma^{2}}
$$

which, upon identifications $\sigma^{2}=2 D t$ and $\Delta\left(\sigma^{2}\right)=2 D \Delta t$, sets an obvious connection with the differential $(\Delta \mathcal{S})(t)$ and thence with the time derivative $\dot{\mathcal{S}}=1 / 2 t$ of the heat kernel differential entropy.

Our previous observations are a special case of more general reasoning. Namely, if we consider a two-parameter $\theta \doteq\left(\theta_{1}, \theta_{2}\right)$ family of densities, then instead of Eq. (15) we would have arrived at

$$
\mathcal{K}(\theta, \theta+\Delta \theta) \simeq \frac{1}{2} \sum_{i, j} \mathcal{F}_{i j} \cdot \Delta \theta_{i} \Delta \theta_{j}
$$

where $i, j,=1,2$ and the Fisher information matrix $\mathcal{F}_{i j}$ is given as follows

$$
\mathcal{F}_{i j}=\int \rho_{\theta} \frac{\partial \ln \rho_{\theta}}{\partial \theta_{i}} \cdot \frac{\partial \ln \rho_{\theta}}{\partial \theta_{j}} d x .
$$

In case of Gaussian densities, labelled by independent parameters $\theta_{1}=\alpha$ and $\theta_{2}=\sigma$ (alternatively $\theta_{2}=\sigma^{2}$ ), the Fisher matrix is diagonal and defined in terms of previous entries $\mathcal{F}_{\alpha}$ and $\mathcal{F}_{\sigma}$ (or $\mathcal{F}_{\sigma^{2}}$ ). 
It is useful to note (c.f. also [9]) that in self-explanatory notation, for two $\theta$ and $\theta^{\prime}$ Gaussian densities there holds:

$$
\mathcal{K}\left(\theta, \theta^{\prime}\right)=\ln \frac{\sigma^{\prime}}{\sigma}+\frac{1}{2}\left(\frac{\sigma^{2}}{{\sigma^{\prime}}^{2}}-1\right)+\frac{1}{2{\sigma^{\prime}}^{2}}\left(\alpha-\alpha^{\prime}\right)^{2}
$$

The first entry in Eq. (23) coincides with the "absolute comparison formula" for Shannon entropies, Eq. (13). However for $\left|\theta^{\prime}-\theta\right| \ll 1$, the second term dominates the first one.

Indeed, let us set $\alpha^{\prime}=\alpha$ and consider $\sigma^{2}=2 D t, \Delta\left(\sigma^{2}\right)=2 D \Delta t$. Then $\mathcal{S}\left(\sigma^{\prime}\right)-\mathcal{S}(\sigma) \simeq \Delta t / 2 t$, while $\mathcal{K}\left(\theta, \theta^{\prime}\right) \simeq(\Delta t)^{2} / 4 t^{2}$. Although, for finite increments $\Delta t$ we have

$$
\mathcal{S}\left(\sigma^{\prime}\right)-\mathcal{S}(\sigma) \simeq \sqrt{\mathcal{K}\left(\theta, \theta^{\prime}\right)} \simeq \frac{\Delta t}{2 t}
$$

the time derivative can be defined exclusively for the differential entropy, $\dot{\mathcal{S}}$, and is meaningless in terms of the Kullback "distance".

Let us mention that no such obstacles arise in the standard cautious use of the relative Kullback entropy $\mathcal{H}_{c}$. Indeed, normally one of the involved densities stands for the stationary reference one $\rho_{\theta^{\prime}}(x) \doteq \rho_{*}(x)$, while another evolves in time $\rho_{\theta}(x) \doteq \rho(x, t), t \in R^{+}$, thence we need:

$$
\mathcal{H}_{c}(t) \doteq-\mathcal{K}\left(\rho_{t} \mid \rho_{*}\right)
$$

In the presence of external forces, the property Eq. (12) may consistently quantify an asymptotic approach towards a minimum corresponding to an invariant (presumed to be unique) probability density of the process. Indeed, by invoking Eq. (7) we realize that

$$
\rho_{*}(x)=\frac{1}{Z} \exp \left(-\frac{V(x)}{k_{B} T}\right)
$$

where $Z=\int \exp \left(-V(x) / k_{B} T\right) d x$, sets the minimum of $\langle\Psi\rangle(t)$ at $\langle\Psi\rangle_{*}=\Psi_{*}=-k_{B} T \ln Z$.

Let us take the above $\rho_{*}(x)$ as a reference density with respect to which the divergence of $\rho(x, t)$ is evaluated in the course of the pertinent Smoluchowski process. This divergence is well quantified by the conditional Kullback entropy $\mathcal{H}_{c}(t)$, where:

$$
\mathcal{H}_{c}(t)=-\int \rho \ln \left(\frac{\rho}{\rho_{*}}\right) d x=\mathcal{S}(t)-\ln Z-\frac{\langle V\rangle}{k_{B} T} .
$$

Consequently, in view of Eqs. (11) and (5), we get

$$
\dot{\mathcal{H}}_{c}=\dot{\mathcal{S}}+\dot{\mathcal{Q}}=(\dot{\mathcal{S}})_{i n} \geq 0
$$

so that $\frac{d}{d t}\langle\Psi\rangle=-\left(k_{B} T\right) \dot{\mathcal{H}}_{c}$. An approach of $\langle\Psi\rangle(t)$ towards the minimum proceeds in the very same rate as this of $\mathcal{H}_{c}(t)$ towards its maximum.

In contrast to $\dot{\mathcal{H}}_{c}$ which is non-negative, we have no growth guarantee for the differential entropy, since the sign of $\dot{\mathcal{S}}$ is unspecified. Nonetheless, the balance between the time rate of entropy 
production/removal and the power release into or out of the environment, is definitely correct and informative.

We have $\dot{\mathcal{S}} \geq-\dot{\mathcal{Q}}$ and surely $\dot{\mathcal{Q}}<0 \rightarrow \dot{\mathcal{S}}>0$. If $\dot{\mathcal{Q}}>0, \dot{\mathcal{S}}$ may take negative values down to the lower bound $-\dot{\mathcal{Q}}$.

It is quite illuminating to exemplify previous considerations by a detailed presentation of the standard one-dimensional Ornstein-Uhlenbeck process. We denote $b(x)=-\gamma x$ with $\gamma>0$ and choose an initial density in the Gaussian form, with the mean value $\alpha_{0}$ and variance $\sigma_{0}^{2}$. The Fokker-Planck evolution Eq. (11) preserves the Gaussian form of $\rho(x, t)$ while modifying the mean value and variance according to $\alpha(t)=\alpha_{0} \exp (-\gamma t)$, and

$$
\sigma^{2}(t)=\sigma_{0}^{2} \exp (-2 \gamma t)+\frac{D}{\gamma}[1-\exp (-2 \gamma t)]
$$

Accordingly, since a unique invariant density has the form $\rho_{*}=\sqrt{\gamma / 2 \pi D} \exp \left(-\gamma x^{2} / 2 D\right)$ we obtain, [9]:

$$
\mathcal{H}_{c}(t)=\exp (-2 \gamma t) \mathcal{H}_{c}\left(\rho_{0}, \rho_{*}\right)=-\frac{\gamma \alpha_{0}^{2}}{2 D} \exp (-2 \gamma t)
$$

while in view of our previous considerations, we have $\mathcal{S}(t)=(1 / 2) \ln \left[2 \pi e \sigma^{2}(t)\right]$ and $\mathcal{F}=1 / \sigma^{2}(t)$. Therefore

$$
\dot{\mathcal{S}}=\frac{2 \gamma\left(D-\gamma \sigma_{0}^{2}\right) \exp (-2 \gamma t)}{D-\left(D-\gamma \sigma_{0}^{2}\right) \exp (-2 \gamma t)}
$$

We observe that $\sigma_{0}^{2}>D / \gamma \rightarrow \dot{\mathcal{S}}<0$, while $\sigma_{0}^{2}<D / \gamma \rightarrow \dot{\mathcal{S}}>0$. In both cases the behavior of the differential entropy is monotonic, although its ultimate growth or decay do critically rely on the choice of $\sigma_{0}^{2}$. Irrespective of $\sigma_{0}^{2}$ the asymptotic value of $\mathcal{S}(t)$ as $t \rightarrow \infty \operatorname{reads}(1 / 2) \ln [2 \pi e(D / \gamma)$.

The differential entropy evolution is anti-correlated with the Fisher measure of the probability localization, since

$$
\dot{\mathcal{F}}=-\frac{\gamma \dot{\mathcal{S}}}{\left[D-\left(D-\gamma \sigma_{0}^{2}\right) \exp (-2 \gamma t)\right]^{2}} .
$$

For all $\sigma_{0}^{2}$ the asymptotic value of $\mathcal{F}$ reads $\gamma / D$.

We have here a direct control of the behavior of the "power release" expression $\dot{\mathcal{Q}}=\dot{\mathcal{H}}_{c}-\dot{\mathcal{S}}$. Since

$$
\dot{\mathcal{H}}_{c}=\left(\gamma^{2} \alpha_{0}^{2} / D\right) \exp (-2 \gamma t)>0
$$

in case of $\dot{\mathcal{S}}<0$ we encounter a continual power removal $\dot{\mathcal{Q}}>0$ into the thermal environment.

In case of $\dot{\mathcal{S}}>0$ the situation is more complicated. For example, if $\alpha_{0}=0$, we can easily check that $\dot{\mathcal{Q}}<0$, i.e. we have the power supply from the environment for all $t \in R^{+}$. More generally, the sign of $\dot{\mathcal{Q}}$ is negative for $\alpha_{0}^{2}<2\left(D-\gamma \sigma_{0}^{2}\right) / \gamma$. If the latter inequality is reversed, the sign of $\dot{\mathcal{Q}}$ is not uniquely specified and suffers a change at a suitable time instant $t_{\text {change }}\left(\alpha_{0}^{2}, \sigma_{0}^{2}\right)$.

Standard notions of thermodynamical entropy are basically not considered in the time domain. However any conceivable idea of "approaching" the state of equilibrium or passing from one such 
state to another (steady) state, surely involves the time dependence and the related non-equilibrium dynamical process. This refers to attempts to give a precise meaning to Boltzmann's H-theorem under non-equilibrium conditions and search for an origin of increasing entropy in terms of model systems. Consult e.g. at this point, both standard motivations and apparent problems encountered in connection with the $H$-theorem and its diffusion process analogues, 2, 8, 7,

Our analysis of simple diffusion-type models indicates that the very notion of entropy is nonuniversal and purpose-dependent. In particular, although the conditional Kullback entropy is often considered as as the only valid "entropy growth" justification, a deeper insight into the underlying physical phenomena (power transfer processes) is available only through the differential (Shannon) entropy notion.

Acknowledgement: The paper has been supported by the Polish Ministry of Scientific Research an Information Technology (solicited) grant No PBZ-MIN-008/P03/2003.

\section{References}

[1] H. Risken, The Fokker-Planck Equation, Springer-Verlag, Berlin, 1989

[2] H. Hasegawa, Progr. Theor. Phys. 57 (1977), 1523-1537

[3] J. M. G. Vilar and J. M. and Rubi, Proc. Nat. Acad. Sci. (NY) 98 (2001), 11081-11084

[4] C. E. Shannon: A mathematical theory of communication, Bell Syst. Techn. J. 27, (1948), 379$423,623-656$

[5] T. M. Cover and J. A. Thomas, Elements of Information Theory, Wiley, NY, 1991

[6] K. Sobczyk, Mechanical Systems and Signal Processing, 15 (2001), 475-498

[7] A. Lasota and M. C. Mackey, Chaos, Fractals and Noise, Springer-Verlag, Berlin, 1994

[8] D-Q. Jiang, M. Qian and M-P. Qian, Mathematical theory of nonequilibrium steady states, LNM vol. 1833, Springer-Verlag, Berlin, 2004

[9] M. C. Mackey and M. Tyran-Kamińska, Effects of noise on entropy evolution, arXiv.org preprint cond-mat/0501092 (2005) 